\title{
The Bloch space for the minimal ball
}

\author{
by \\ G. Mengotti (Fribourg)
}

\begin{abstract}
We introduce the Bloch space for the minimal ball and we prove that this space can be identified with the dual of a certain analytic space which is strongly related to the Bergman theory on the minimal ball.
\end{abstract}

1. Introduction and main result. Let $\mathbb{B}_{*}$ be the domain in $\mathbb{C}^{n}, n \geq 2$, defined by

$$
\mathbb{B}_{*}:=\left\{z \in \mathbb{C}^{n}:|z|^{2}+|z \bullet z|<1\right\},
$$

where $z \bullet w:=\sum_{j=1}^{n} z_{j} w_{j}$ for $z$ and $w$ in $\mathbb{C}^{n}$. This is the unit ball with respect to the norm

$$
N_{*}(z):=\sqrt{|z|^{2}+|z \bullet z|}, \quad z \in \mathbb{C}^{n} .
$$

The norm $N:=N_{*} / \sqrt{2}$ was introduced by Hahn and Pflug [HP], and was shown to be the smallest norm in $\mathbb{C}^{n}$ that extends the euclidian norm in $\mathbb{R}^{n}$ under certain restrictions. The automorphism group of $\mathbb{B}_{*}$ is compact and its identity component is $\operatorname{Aut}_{\mathcal{O}}^{0}\left(\mathbb{B}_{*}\right)=S^{1} \cdot \mathrm{SO}(n, \mathbb{R})$, where the $S^{1}$-action is diagonal and the $\mathrm{SO}(n, \mathbb{R})$-action is the matrix multiplication (see $[\mathrm{K}]$ or $[\mathrm{OY}])$. The ball $\mathbb{B}_{*}$ is a nonhomogeneous domain with singular boundary consisting of all its boundary points $z$ that satisfy $z \bullet z=0$. The regular part of the boundary of $\mathbb{B}_{*}$ consists of strictly pseudoconvex points. The Bergman theory on the minimal ball, developed in $[\mathrm{MY}]$, showed the importance of this singularity. $\mathbb{B}_{*}$ was also used to construct counter-examples to the Lu Qi-Keng conjecture [PY]. This makes the analysis on the minimal ball very interesting. Furthermore E. H. Youssfi recently proved $[\mathrm{Y}]$ that the methods used in [MY] can be extended to a more general class of domains in $\mathbb{C}^{n}$ containing the minimal ball, the unit ball and the complex

2000 Mathematics Subject Classification: Primary 32C37, 46E30; Secondary 32A36, $47 \mathrm{G} 10$.

Key words and phrases: minimal ball, dual space, reproducing kernel.

Supported by the Fonds National Suisse de la Recherche Scientifique; Grant $\mathrm{N}^{\mathrm{O}} 20$ 56811.99 . 
ellipsoids. The study of $\mathbb{B}_{*}$ seems to be a good way to understand a much wider case.

Let $v$ be the normalized Lebesgue measure on $\mathbb{B}_{*}$. For $p \geq 1$ let $L^{p}\left(\mathbb{B}_{*}\right.$, $\left.|z \bullet z|^{(p-2) / 2} d v\right)$ be the Banach space of all functions on $\mathbb{B}_{*}$ that are $L^{p}$-integrable with respect to the measure $|z \bullet z|^{(p-2) / 2} d v(z)$. We denote by $\mathcal{A}^{p}\left(\mathbb{B}_{*}\right)$ the space of all holomorphic functions on $\mathbb{B}_{*}$ which are in the space $L^{p}\left(\mathbb{B}_{*}\right.$, $\left.|z \bullet z|^{(p-2) / 2} d v\right)$. The spaces $\mathcal{A}^{p}\left(\mathbb{B}_{*}\right)$ appear naturally in Bergman theory associated to the minimal ball (see $[\mathrm{MY}]$ ). It is known that these spaces furnished with the norm of $L^{p}\left(\mathbb{B}_{*},|z \bullet z|^{(p-2) / 2} d v\right)$ are Banach spaces (see Lemma 4.1 of $[\mathrm{MY}])$.

In $[\mathrm{M}]$ we proved that for $p>1$ the dual space of $\mathcal{A}^{p}\left(\mathbb{B}_{*}\right)$ can be identified with $\mathcal{A}^{q}\left(\mathbb{B}_{*}\right)$ where $1 / p+1 / q=1$. Here we are interested in the case $p=1$. For the unit ball it is well known that the dual space of the Bergman space of order 1 is the Bloch space (see for instance [A], [Z2] for the one-dimensional case and [Ch], [Tim1], [Tim2] for generalizations). There are also various results for other domains (see for instance $[\mathrm{B}],[\mathrm{Z1}]$, [Tem] for bounded symetric domains, and $[\mathrm{Co}],[\mathrm{L}],[\mathrm{KM}]$ for strictly pseudoconvex domains). But these results are not applicable in the case of $\mathbb{B}_{*}$.

Usually the definition of the Bloch space depends on the gradient growth. But Timoney showed that we can also characterize it using the radial derivative (see Theorem 4.10 of [Tim1]). In the case of $\mathbb{B}_{*}$ we will use the following definition.

Let $\mathcal{B}\left(\mathbb{B}_{*}\right)$ be the space of all holomorphic functions $f$ on $\mathbb{B}_{*}$ such that

$$
\|f\|_{\mathcal{B}\left(\mathbb{B}_{*}\right)}=|f(0)|+\sup _{\mathbb{B}_{*}} \frac{|R f(z)|}{|z|}|z \bullet z|^{1 / 2}\left(1-N_{*}^{2}(z)\right)<\infty,
$$

where

$$
R f(z)=\sum_{i=1}^{n} z_{i} \frac{\partial f}{\partial z_{i}}(z) \quad \text { for all } f \in \operatorname{Hol}\left(\mathbb{B}_{*}\right) \text { and } z \in \mathbb{B}_{*} .
$$

$R f$ is called the radial derivative of $f$. We easily see that $\|\cdot\|_{\mathcal{B}\left(\mathbb{B}_{*}\right)}$ defines a norm on $\mathcal{B}\left(\mathbb{B}_{*}\right)$. We will prove that $\mathcal{B}\left(\mathbb{B}_{*}\right)$ is a Banach space and that, for all $p \geq 1, \mathcal{B}\left(\mathbb{B}_{*}\right) \subset \mathcal{A}^{p}\left(\mathbb{B}_{*}\right)$ (see Corollary 4.2 ).

Our main result is the following

Theorem 1.1. The dual of the space $\mathcal{A}^{1}\left(\mathbb{B}_{*}\right)$ can be identified with $\mathcal{B}\left(\mathbb{B}_{*}\right)$. More precisely, there is a bounded bilinear complex form $\Lambda_{\mathbb{B}_{*}}$ on $\mathcal{A}^{1}\left(\mathbb{B}_{*}\right) \times \mathcal{B}\left(\mathbb{B}_{*}\right)$ such that every bounded linear functional on $\mathcal{A}^{1}\left(\mathbb{B}_{*}\right)$ is of the form

$$
f \mapsto \Lambda_{\mathbb{B}_{*}}(f, g)
$$

for some unique $g \in \mathcal{B}\left(\mathbb{B}_{*}\right)$. Furthermore the norm of the linear functional on $\mathcal{A}^{1}\left(\mathbb{B}_{*}\right)$ is equivalent to the norm of $g$ in $\mathcal{B}\left(\mathbb{B}_{*}\right)$, i.e. there is a positive 
constant $\tau$ such that

$$
\tau\|g\|_{\mathcal{B}\left(\mathbb{B}_{*}\right)} \leq\left\|\Lambda_{\mathbb{B}_{*}}(\cdot, g)\right\| \leq \tau^{-1}\|g\|_{\mathcal{B}\left(\mathbb{B}_{*}\right)}
$$

for all $g \in \mathcal{B}\left(\mathbb{B}_{*}\right)$.

This work was done at the "Institut de Mathématiques de Fribourg (Switzerland)". I wish to thank the National Swiss Foundation for supporting me and all the members of the institute for their friendly welcome. Finally I dedicate this paper to my family.

\section{Preliminaries}

Notation 2.1. In this paper we denote by $\operatorname{Hol}(X)$ the set of all holomorphic functions on $X$ where $X$ is a complex manifold.

The domain $\mathbb{B}_{*}$ is strongly related to the hypersurface $\mathbb{M}$ of the unit ball in $\mathbb{C}^{n+1}$ defined by

$$
\mathbb{M}=\left\{z \in \mathbb{C}^{n+1} \backslash\{0\}: z \bullet z=0,|z|<1\right\},
$$

where $z \bullet w:=\sum_{j=1}^{n} z_{j} w_{j}$ for $z$ and $w$ in $\mathbb{C}^{n+1}$. Let $\operatorname{Pr}: \mathbb{C}^{n+1} \rightarrow \mathbb{C}^{n}$ be defined by $\operatorname{Pr}\left(z_{1}, \ldots, z_{n}, z_{n+1}\right)=\left(z_{1}, \ldots, z_{n}\right)$ and $F=\operatorname{Pr}_{\mid \mathbb{M}}$. Then $F: \mathbb{M} \rightarrow \mathbb{B}_{*} \backslash\{0\}$ is a proper holomorphic mapping of degree 2 . We denote by $W$ the branching locus of $F$. The image $F(W)$ is an analytic subset of $\mathbb{B}_{*} \backslash\{0\}$. We set $V:=F(W) \cup\{0\}$. The local inverses $\phi$ and $\psi$ of $F$ are given for $z \in \mathbb{B}_{*} \backslash V$ by

$$
\phi(z)=(z, i \sqrt{z \bullet z}), \quad \psi(z)=(z,-i \sqrt{z \bullet z}) .
$$

Set

$$
\mathbb{H}=\left\{z \in \mathbb{C}^{n+1} \backslash\{0\}: z \bullet z=0\right\} .
$$

It was proved in [OPY] that there is a unique (up to a multiplicative constant) $\mathrm{SO}(n+1, \mathbb{C})$-invariant holomorphic form $\alpha$ on $\mathbb{H}$. The restriction of this form to $\mathbb{H} \cap(\mathbb{C} \backslash\{0\})^{n+1}$ is given by

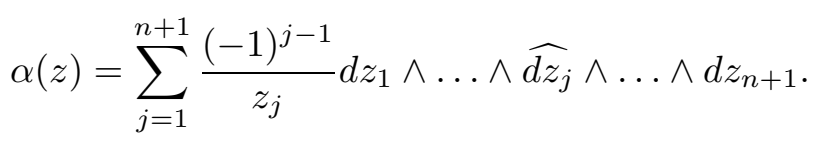

Definition 2.2. 1. $L^{1}(\mathbb{M})$ is the space of measurable complex functions on $\mathbb{M}$ such that

$$
\|f\|_{L^{1}(\mathbb{M})}=\int_{\mathbb{M}}|f(z)| \frac{\alpha(z) \wedge \overline{\alpha(z)}}{C}<\infty \quad \text { where } \quad C:=(-1)^{n(n+1) / 2}(2 i)^{n} .
$$

2. $\mathcal{A}^{1}(\mathbb{M})$ is the subspace of all holomorphic functions in $L^{1}(\mathbb{M})$.

All along this note we will use the following important operator. 
Definition 2.3. Let $f: \mathbb{B}_{*} \rightarrow \mathbb{C}$ be a measurable function. We define $T f$ by

$$
(T f)(z):=\frac{1}{2(n+1)^{2}} z_{n+1}(f \circ F)(z)
$$

for all $z=\left(z_{1}, \ldots, z_{n+1}\right) \in \mathbb{M}$.

In [MY] (Lemma 4.1) we proved the following.

LEMma 2.4. The operator $T$ is an isometry from $L^{1}\left(\mathbb{B}_{*}, d v(z) /|z \bullet z|^{1 / 2}\right)$ into $L^{1}(\mathbb{M})$. More precisely, we have

$$
\int_{\mathbb{M}}|(T f)(z)| \frac{\alpha(z) \wedge \overline{\alpha(z)}}{C}=\int_{\mathbb{B}_{*}}|f(w)| \cdot|w \bullet w|^{-1 / 2} d v(w) .
$$

In addition, the image $\mathcal{E}^{1}(\mathbb{M})$ of $\mathcal{A}^{1}\left(\mathbb{B}_{*}\right)$ under $T$ is a closed proper subspace of $\mathcal{A}^{1}(\mathbb{M})$ and $T$ is a unitary operator from $\mathcal{A}^{1}\left(\mathbb{B}_{*}\right)$ onto $\mathcal{E}^{1}(\mathbb{M})$. In particular, $\mathcal{A}^{1}\left(\mathbb{B}_{*}\right)$ is a Banach space.

REMARK 2.5. In fact we have

$$
\mathcal{E}^{1}(\mathbb{M})=L^{1}(\mathbb{M}) \cap T\left(\operatorname{Hol}\left(\mathbb{B}_{*}\right)\right) .
$$

3. An intermediate result. In order to prove the main theorem on $\mathbb{B}_{*}$ we will establish an intermediate result on $\mathbb{M}$. More precisely we will find the dual space of $\mathcal{E}^{1}(\mathbb{M})$ (see Theorem 3.14). For this purpose we must first introduce some new analytic spaces.

3.1. Other analytic spaces on $\mathbb{M}$

Definition 3.1. Let $f$ be a holomorphic function on $\mathbb{M}$ and $z \in \mathbb{M}$.

1. As usual $f_{z}$ denotes the slice function defined by

$$
f_{z}(\tau)=f(\tau z)
$$

for all $\tau \in \mathbb{C}$ such that $0<|\tau|<1 /|z|$.

2. We set

$$
R f(z)=\left.\frac{\partial f_{z}}{\partial \tau}\right|_{\tau=1} .
$$

$R f$ is called the radial derivative of $f$.

Lemma 3.2. Let $f \in \operatorname{Hol}(\mathbb{M})$.

1. $f$ can be uniquely extended to the complex hypersurface $\mathbb{M} \cup\{0\}$ so that we can define $f(0)$.

2. We have

$$
f(z)=f(0)+\int_{0}^{1} \frac{R f(t z)}{t} d t .
$$


Proof. Let $\mathbb{B}_{n+1}$ be the unit ball in $\mathbb{C}^{n+1}$. By the proof of Lemma 3.1 of $[\mathrm{MY}]$ there is a function $g \in \operatorname{Hol}\left(\mathbb{B}_{n+1}\right)$ such that $g_{\mid \mathbb{M}}=f$.

1. Thus $\lim _{z \rightarrow 0, z \in \mathbb{M}} f(z)$ exists and we can define $f(0)$.

2. For all $z \in \mathbb{B}_{n+1}$ we can write $g(z)=g(0)+\int_{0}^{1} \frac{\partial g_{z}}{\partial t}(t) d t$. But by Definition 3.1 it is also clear that for all $z \in \mathbb{M}$,

$$
\frac{\partial g_{z}}{\partial t}(t)=\frac{\partial f_{z}}{\partial t}(t)=\frac{R f(t z)}{t}
$$

Definition 3.3. $\mathcal{B}(\mathbb{M})$ is the space of all holomorphic functions such that

$$
\|f\|_{\mathcal{B}(\mathbb{M})}=|f(0)|+\sup _{z \in \mathbb{M}} \frac{|R f(z)|}{|z|}\left(1-|z|^{2}\right)<\infty .
$$

From Lemma 3.2 it follows that $\|f\|_{\mathcal{B}(\mathbb{M})}$ is a norm on $\mathcal{B}(\mathbb{M})$.

Lemma 3.4. Let $f \in \mathcal{B}(\mathbb{M})$.

1. We have

$$
\sup _{z \in \mathbb{M}}|f(z)|\left(1-|z|^{2}\right) \leq 2\|f\|_{\mathcal{B}(\mathbb{M})} .
$$

2. For all $p>0, f \in L^{p}(\mathbb{M})$. The inclusion of $\mathcal{B}(\mathbb{M})$ in $L^{p}(\mathbb{M})$ is continuous.

Proof. 1. By Lemma 3.2 we have

$$
\begin{aligned}
|f(z)| & \leq|f(0)|+\int_{0}^{1}\left|\frac{R f(t z)}{t}\right| d t \\
& \leq\|f\|_{\mathcal{B}(\mathbb{M})}+\|f\|_{\mathcal{B}(\mathbb{M})} \int_{0}^{1} \frac{t|z|}{t\left(1-(t|z|)^{2}\right)} d t \\
& \leq\|f\|_{\mathcal{B}(\mathbb{M})}+\|f\|_{\mathcal{B}(\mathbb{M})} \int_{0}^{|z|} \frac{d u}{1-t^{2}} d t \\
& \leq 2\|f\|_{\mathcal{B}(\mathbb{M})}\left(1-\ln \left(1-|z|^{2}\right)\right) .
\end{aligned}
$$

But it is clear that

$$
1-\ln \left(1-|z|^{2}\right) \leq \frac{1}{1-|z|^{2}}
$$

on $\mathbb{M}$, which completes the proof of assertion 1 .

2. Now we fix $p^{\prime}>p$. Then there is a positive constant $M_{p^{\prime}}$ such that

$$
1-\ln \left(1-|z|^{2}\right) \leq \frac{M_{p^{\prime}}}{\left(1-|z|^{2}\right)^{1 / p^{\prime}}}
$$

on $\mathbb{M}$. But integration in polar coordinates (see Lemma 2.1 of $[\mathrm{MY}]$ ) proves that the function $M_{p^{\prime}} /\left(1-|z|^{2}\right)^{1 / p^{\prime}}$ is in $L^{p}(\mathbb{M})$ since $p / p^{\prime}<1$. This leads to assertion 2 . 
DeFinition 3.5.

$$
\mathcal{E}(\mathbb{M})=\mathcal{B}(\mathbb{M}) \cap T\left(\operatorname{Hol}\left(\mathbb{B}_{*}\right)\right) .
$$

Lemma 3.6. Furnished with the Bloch norm, $\mathcal{B}(\mathbb{M})$ and $\mathcal{E}(\mathbb{M})$ are Banach spaces.

Proof. The fact that $\mathcal{B}(\mathbb{M})$ is a Banach space is a classical consequence of Lemma 3.4. Then it suffices to show that $\mathcal{E}(\mathbb{M})$ is a closed subset of $\mathcal{B}(\mathbb{M})$. Let $\left(f_{k}\right)_{k \in \mathbb{N}} \subset \mathcal{E}(\mathbb{M})$ tend to $f$ in $\mathcal{B}(\mathbb{M})$. Again by Lemma 3.4 we know that $f_{k} \rightarrow f$ in $L^{1}(\mathbb{M})$. But by Remark $2.5, f_{k} \in \mathcal{E}^{1}(\mathbb{M})$, and by Lemma $2.4, \mathcal{E}^{1}(\mathbb{M})$ is a closed subset of $L^{1}(\mathbb{M})$ so that $f \in \mathcal{E}^{1}(\mathbb{M})$. In particular $f \in T\left(\operatorname{Hol}\left(\mathbb{B}_{*}\right)\right)$.

We will use the following space.

DeFinition 3.7.

$$
\mathcal{E}^{\infty}(\mathbb{M})=L^{\infty}(\mathbb{M}) \cap T\left(\operatorname{Hol}\left(\mathbb{B}_{*}\right)\right) .
$$

Lemma 3.8. $\mathcal{E}^{\infty}(\mathbb{M})$ is dense in $\mathcal{E}^{1}(\mathbb{M})$.

Proof. By usual methods we can show that if $f \in L^{1}(\mathbb{M})$ then $f_{r} \rightarrow f$ in $L^{1}(\mathbb{M})$ as $r \rightarrow 1^{-}$. But if $f \in \mathcal{E}^{1}(\mathbb{M})$ then $f_{r} \in \mathcal{E}^{\infty}(\mathbb{M})$.

3.2. The dual space of $\mathcal{E}^{1}(\mathbb{M})$

Notation 3.9. 1. $\widetilde{P}$ denotes the orthogonal projection from $L^{2}(\mathbb{M})$ onto $\mathcal{E}^{2}(\mathbb{M})$ (which is a closed subspace of $L^{2}(\mathbb{M})$ by Lemma 4.1 of $[\mathrm{MY}]$ ).

2. $K_{\mathbb{B}_{*}}$ denotes the Bergman kernel of $\mathbb{B}_{*}$ (see $[\mathrm{OPY}]$ or $[\mathrm{MY}]$ ).

We recall the following (see Lemma 2 of $[\mathrm{M}]$ ).

Lemma 3.10. The projection $\widetilde{P}$ is an integral operator induced by the kernel

$$
\widetilde{K}(z, w)=\frac{1}{2(n+1)^{2}} z_{n+1} \bar{w}_{n+1} K_{\mathbb{B}_{*}}(F(z), F(w))
$$

for all $z$ and $w$ in $\mathbb{M}$.

Lemma 3.11. The projection $\widetilde{P}$ is a well defined bounded operator from $L^{\infty}(\mathbb{M})$ into $\mathcal{E}(\mathbb{M})$.

Proof. In this proof $M$ will denote a constant depending only on $n$ which may differ at each appearance. Let $f \in L^{\infty}(\mathbb{M})$. Then $\widetilde{P} f \in \mathcal{E}^{2}(\mathbb{M})$ so that $\widetilde{P} f \in T(\operatorname{Hol}(\mathbb{M}))$. So we must only prove that $\widetilde{P} f \in \mathcal{B}(\mathbb{M})$. We have

$$
\widetilde{P} f(z)=\int_{\mathbb{M}} f(w) \widetilde{K}(z, w) \frac{\alpha(w) \wedge \overline{\alpha(w)}}{C} .
$$

Thus

$$
R(\widetilde{P} f)(z)=\int_{\mathbb{M}} f(w) R(z \mapsto \widetilde{K}(z, w)) \frac{\alpha(w) \wedge \overline{\alpha(w)}}{C}
$$


Using Lemma 4.2 of $[\mathrm{MY}]$ we find that, for all $z$ and $w$ in $\mathbb{M}$,

$$
\widetilde{K}(z, w)=M\left[K_{\mathbb{M}}(z, w)-K_{\mathbb{M}}(z, A(w))\right]
$$

where $A$ is the transformation of $\mathbb{C}^{n+1}$ defined by

$$
A\left(z_{1}, \ldots, z_{n}, z_{n+1}\right)=\left(z_{1}, \ldots, z_{n},-z_{n+1}\right)
$$

and $K_{\mathbb{M}}$ is the Bergman kernel of $\mathbb{M}$ with respect to the volume form $\alpha(w) \wedge$ $\overline{\alpha(w)} / C$ (see Theorem 3.2 of $[\mathrm{MY}]$ ). Thus

$$
R(z \mapsto \widetilde{K}(z, w))=M\left[R\left(z \mapsto K_{\mathbb{M}}(z, w)\right)-R\left(z \mapsto K_{\mathbb{M}}(z, A(w))\right)\right] .
$$

By Theorem 3.2 of $[\mathrm{MY}]$ and a little computation we obtain

$$
R\left(z \mapsto K_{\mathbb{M}}(z, w)\right)=M \frac{(z \bullet \bar{w})[1+z \bullet \bar{w}]}{(1-z \bullet \bar{w})^{n+2}} .
$$

Hence

$$
\left|R\left(z \mapsto K_{\mathbb{M}}(z, w)\right)\right| \leq M \frac{|z|}{|1-z \bullet \bar{w}|^{n+2}} .
$$

Then Lemma 5.1 of $[\mathrm{MY}]$ gives

$$
|R(\widetilde{P} f)(z)| \leq M \frac{|z| \cdot\|f\|_{\infty}}{1-|z|^{2}} .
$$

Since $\widetilde{P} f(0)=0$, this exactly says that

$$
\|\widetilde{P} f\|_{\mathcal{B}(\mathbb{M})} \leq M\|f\|_{\infty}
$$

Definition 3.12. 1. For $f \in \operatorname{Hol}(\mathbb{M})$ and $z \in \mathbb{M}$ we put

$$
Q f(z)=\left(1-|z|^{2}\right)(n f(z)+R f(z)) .
$$

2. For all functions $f$ and $g$ measurable in $\mathbb{M}$ we set

$$
\langle f, g\rangle_{\mathbb{M}}=\int_{\mathbb{M}} f(w) \overline{g(w)} \frac{\alpha(w) \wedge \overline{\alpha(w)}}{C}
$$

provided the above integral is defined.

Then we have the following

LEMma 3.13. 1. $Q$ is a well defined bounded operator from $\mathcal{B}(\mathbb{M})$ into $L^{\infty}(\mathbb{M})$.

2. For all $h \in \mathcal{A}(\mathbb{M}):=L^{\infty}(\mathbb{M}) \cap \operatorname{Hol}(\mathbb{M})$ and $g \in \mathcal{B}(\mathbb{M})$ we have

$$
\langle h, g\rangle_{\mathbb{M}}=\langle h, Q g\rangle_{\mathbb{M}} \text {. }
$$

Proof. 1. Let $f \in \mathcal{B}(\mathbb{M})$ and $z \in \mathbb{M}$. We have

$$
|Q f(z)| \leq n\left(1-|z|^{2}\right)|f(z)|+\left(1-|z|^{2}\right)|R f(z)|
$$

and by Lemma 3.4 we obtain

$$
|Q f(z)| \leq(2 n+1)\|f\|_{\mathcal{B}(\mathbb{M})} .
$$


2. Firstly we note that by Lemma 3.4 and by the first assertion the above scalar products are well defined. Then recall that by the proof of Theorem 3.2 of $[\mathrm{MY}]$ we know that if $h \in \mathcal{A}(\mathbb{M})$ then

$$
h=\sum_{k=0}^{\infty} p_{k}
$$

in $L^{2}(\mathbb{M})$ where $p_{k} \in \mathcal{P}_{k}(\mathbb{M})$, the set of homogeneous polynomials of degree $k$ on $\mathbb{M}$. So we need only verify the formula for $h=p_{k}$ and $g \in \mathcal{B}(\mathbb{M})$. But we also know that the sum is orthogonal in $L^{2}(\mathbb{M})$ and that $g \in L^{2}(\mathbb{M})$. Thus it suffices to prove that

$$
\left\langle p_{k}, q_{k}\right\rangle_{\mathbb{M}}=\left\langle p_{k}, Q q_{k}\right\rangle_{\mathbb{M}}
$$

for all $p_{k}$ and $q_{k}$ in $\mathcal{P}_{k}(\mathbb{M})$. But $R q_{k}=k q_{k}$ so that

$$
Q\left(q_{k}\right)(z)=(n+k)\left(1-|z|^{2}\right) q_{k}(z) .
$$

Using integration in polar coordinates (see Lemma 2.1 of [MY]) we can prove the previous equality and the result follows.

Now we can prove the intermediate theorem on $\mathbb{M}$.

THeOREm 3.14. The dual of the space $\mathcal{E}^{1}(\mathbb{M})$ can be identified with $\mathcal{E}(\mathbb{M})$. More precisely, there is a bounded bilinear complex form $\Lambda_{\mathbb{M}}$ on $\mathcal{E}^{1}(\mathbb{M}) \times$ $\mathcal{E}(\mathbb{M})$ such that every bounded linear functional on $\mathcal{E}^{1}(\mathbb{M})$ is of the form $\Lambda_{\mathbb{M}}(\cdot, g)$ for some unique $g \in \mathcal{B}\left(\mathbb{B}_{*}\right)$. $\Lambda_{\mathbb{M}}$ is defined for all $h \in \mathcal{E}^{\infty}(\mathbb{M})$ and $g \in \mathcal{E}(\mathbb{M})$ by

$$
\Lambda_{\mathbb{M}}(h, g)=\langle h, g\rangle_{\mathbb{M}}
$$

Furthermore the norms of $g$ and $\Lambda_{\mathbb{M}}(\cdot, g)$ are equivalent.

Proof. Firstly by Lemma 3.13 we can write, for all $h \in \mathcal{E}^{\infty}(\mathbb{M})$ and $g \in \mathcal{E}(\mathbb{M})$,

$$
\left|\langle h, g\rangle_{\mathbb{M}}\right|=\left|\langle h, Q g\rangle_{\mathbb{M}}\right| \leq\|h\|_{1}\|Q g\|_{\infty} \leq\|h\|_{1}\|Q\| \cdot\|g\|_{\mathcal{B}(\mathbb{M})} .
$$

So $\Lambda_{\mathbb{M}}$ is bounded on $\mathcal{E}^{\infty}(\mathbb{M}) \times \mathcal{E}(\mathbb{M})$. By Lemma $3.8, \Lambda_{\mathbb{M}}$ can be uniquely extended to a bounded bilinear form on $\mathcal{E}^{1}(\mathbb{M}) \times \mathcal{E}(\mathbb{M})$. Lemma 3 of $[\mathrm{M}]$ shows that the $g$ as in the statement is unique.

Now let $L$ be a bounded linear functional on $\mathcal{E}^{1}(\mathbb{M})$. By the HahnBanach theorem, $L$ can be extended to a bounded linear functional $\widetilde{L}$ on $L^{1}(\mathbb{M})$ satisfying $\|L\|=\|\widetilde{L}\|$ and by the Riesz representation theorem we can find a function $f \in L^{\infty}(\mathbb{M})$ such that

$$
\widetilde{L}(h)=\int_{\mathbb{M}} h(z) \overline{f(z)} \frac{\alpha(z) \wedge \overline{\alpha(z)}}{C}
$$


for all $h \in L^{1}(\mathbb{M})$ and $\|\widetilde{L}\|=\|f\|_{\infty}$. By Lemma 3.11 the function $g=\widetilde{P}(f)$ belongs to $\mathcal{E}(\mathbb{M})$ and

$$
\|g\|_{\mathcal{E}(\mathbb{M})} \leq\|\widetilde{P}\| \cdot\|f\|_{\infty}=\|\widetilde{P}\| \cdot\|L\| .
$$

Then for all $h \in \mathcal{E}^{\infty}(\mathbb{M})$ we have

$$
\begin{aligned}
\int_{\mathbb{M}} h(z) \overline{g(z)} \frac{\alpha(z)}{C} & \wedge \overline{\alpha(z)} \\
C & \int_{\mathbb{M}} h(z) \overline{\widetilde{P}(f)} \frac{\alpha(z) \wedge \overline{\alpha(z)}}{C} \\
& =\int_{\mathbb{M}} h(z) \overline{\left(\int_{\mathbb{M}} \widetilde{K}(z, w) f(w) \frac{\alpha(w) \wedge \overline{\alpha(w)}}{C}\right)} \frac{\alpha(z) \wedge \overline{\alpha(z)}}{C} \\
& =\int_{\mathbb{M}} \overline{f(w)}\left(\int_{\mathbb{M}} \widetilde{K}(w, z) h(z) \frac{\alpha(z) \wedge \overline{\alpha(z)}}{C}\right) \frac{\alpha(w) \wedge \overline{\alpha(w)}}{C} \\
& =\langle h, f\rangle=\widetilde{L}(h)=L(h) .
\end{aligned}
$$

Note that the proof of Lemma 4 of $[\mathrm{MY}]$ enables us to use Fubini's theorem. The equivalence of the norms is clear.

4. Proof of the main theorem. To prove the main theorem we need the next lemma and its corollary.

Lemma 4.1. Let $g \in \operatorname{Hol}(\mathbb{M})$. For all $z \in \mathbb{M}$ set $\widetilde{T} g(z)=z_{n+1} g(z)$ and define

$$
\begin{aligned}
& \widetilde{S}(g)=\sup _{z \in \mathbb{M}} \frac{|R(\widetilde{T} g)(z)|}{|z|}\left(1-|z|^{2}\right), \\
& S(g)=|g(0)|+\sup _{z \in \mathbb{M}} \frac{|R(g)(z)|}{|z|}\left(1-|z|^{2}\right)\left|z_{n+1}\right| .
\end{aligned}
$$

Then there is a constant $\gamma$ independent of $g$ such that

$$
\gamma^{-1} \widetilde{S}(g) \leq S(g) \leq \gamma \widetilde{S}(g) .
$$

Proof. First of all note that

$$
R(\widetilde{T} g)(z)=z_{n+1}[g(z)+R g(z)] \quad \text { for all } z \in \mathbb{M} .
$$

Then we recall that $g=\sum_{k=0}^{\infty} p_{k}$ for some $p_{k} \in \mathcal{P}_{k}$ as in the proof of Lemma 3.13. Thus for all $z \in \mathbb{M}$ we can write

$$
R g(z)=\sum_{k=0}^{\infty} k p_{k}(z), \quad \widetilde{T} g(z)=\sum_{k=0}^{\infty} z_{n+1} p_{k}(z)
$$

and so

$$
z_{n+1} R g(z)=\sum_{k=0}^{\infty} z_{n+1} k p_{k}(z) .
$$


Now suppose that

$$
\widetilde{S}(g)=\sup _{z \in \mathbb{M}}|R(\widetilde{T} g)(z)|\left(1-|z|^{2}\right)<\infty .
$$

By (1) we can write

$$
S(g)-|g(0)| \leq \widetilde{S}(g)+\sup _{z \in \mathbb{M}} \frac{|\widetilde{T} g(z)|}{|z|}\left(1-|z|^{2}\right) .
$$

So it suffices to study the last supremum. Let $z \in \partial \mathbb{M}$ where

$$
\partial \mathbb{M}=\left\{z \in \mathbb{C}^{n+1}: z \bullet z=0,|z|=1\right\} .
$$

The slice function $(R(\widetilde{T} g))_{z}$ is holomorphic on $\mathbb{D}$, the unit disc in $\mathbb{C}$. For all $\zeta \in \mathbb{D}$, we have

$$
(R(\widetilde{T} g))_{z}(\zeta)=\sum_{k=0}^{\infty}(k+1)\left[z_{n+1} p_{k}(z)\right] \zeta^{k+1}=\zeta \sum_{k=0}^{\infty}(k+1) a_{k}(z) \zeta^{k}
$$

with $a_{k}(z)=z_{n+1} p_{k}(z)$. But by $(2)$ we have

$$
\frac{\left|(R(\widetilde{T} g))_{z}(\zeta)\right|}{|\zeta|} \leq \frac{\widetilde{S}(g)}{1-|\zeta|^{2}}
$$

Thus for all $k \in \mathbb{N}$ we get

$$
\left|(k+1) a_{k}(z)\right| \leq \widetilde{S}(g) e(k+1) .
$$

Therefore we have, for all $z \in \partial \mathbb{M}$ and $k \in \mathbb{N}$,

$$
\left|a_{k}(z)\right| \leq \widetilde{S}(g) e .
$$

Note that, in particular, $\left|a_{0}(z)\right|=\left|z_{n+1} g(0)\right| \leq \widetilde{S}(g)$ e for all $z \in \partial \mathbb{M}$. For $z=(1 / \sqrt{2}, 0, \ldots, 0, i / \sqrt{2})$ this gives

$$
|g(0)| \leq \sqrt{2} \widetilde{S}(g) e .
$$

We also have

$$
\frac{(\widetilde{T} g)_{z}(\zeta)}{\zeta}=\sum_{k=0}^{\infty} a_{k} \zeta^{k}
$$

for $z \in \partial \mathbb{M}$ and $\zeta \in \mathbb{D}$. Hence

$$
\left|\frac{(\widetilde{T} g)_{z}(\zeta)}{\zeta}\right| \leq \widetilde{S}(g) e \sum_{k=0}^{\infty}|\zeta|^{k} \leq \frac{2 \widetilde{S}(g) e}{1-|\zeta|^{2}}
$$

So we get

$$
\sup _{z \in \mathbb{M}} \frac{|\widetilde{T} g(z)|}{|z|}\left(1-|z|^{2}\right) \leq 2 \widetilde{S}(g) e .
$$

This combined with (3) and (4) shows that there exists $\gamma>0$ such that $S(g) \leq \gamma \widetilde{S}(g)$.

We use the same method to establish the other inequality. 
Corollary 4.2. $T$ is a linear isomorphism from $\mathcal{B}\left(\mathbb{B}_{*}\right)$ onto $\mathcal{E}(\mathbb{M})$ and there is a constant $\delta$ such that for all $f \in \mathcal{B}\left(\mathbb{B}_{*}\right)$,

$$
\delta^{-1}\|f\|_{\mathcal{B}\left(\mathbb{B}_{*}\right)} \leq\|T f\|_{\mathcal{E}(\mathbb{M})} \leq \delta\|f\|_{\mathcal{B}\left(\mathbb{B}_{*}\right)} .
$$

In particular $\mathcal{B}\left(\mathbb{B}_{*}\right)$ is a Banach space and, for all $p \geq 1, \mathcal{B}\left(\mathbb{B}_{*}\right) \subset \mathcal{A}^{p}\left(\mathbb{B}_{*}\right)$.

Proof. In fact the radial derivative of holomorphic functions on $\mathbb{B}_{*}$ can be defined using slice functions as in Definition 3.1 for holomorphic functions on $\mathbb{M}$. This fact, the previous lemma and the equivalence of the norms $|\cdot|$ and $N_{*}$ lead to the desired inequalities. This implies that $T$ is a linear isomorphism from $\mathcal{B}\left(\mathbb{B}_{*}\right)$ onto $\mathcal{E}(\mathbb{M})$ and that $\mathcal{B}\left(\mathbb{B}_{*}\right)$ is a Banach space. Then the inclusion $\mathcal{B}\left(\mathbb{B}_{*}\right) \subset \mathcal{A}^{p}\left(\mathbb{B}_{*}\right)$ follows from Lemma 4.1 of $[\mathrm{MY}]$ and Lemma 3.4.

Now we can prove the main result. Consider the bilinear form $\Lambda_{\mathbb{B}_{*}}$ defined on $\mathcal{A}^{1}\left(\mathbb{B}_{*}\right) \times \mathcal{B}\left(\mathbb{B}_{*}\right)$ by

$$
(f, g) \mapsto \Lambda_{\mathbb{B}_{*}}(f, g)=\Lambda_{\mathbb{M}}(T f, T g) .
$$

This mapping is well defined and bounded by Lemma 2.4, the previous corollary and Theorem 3.14. Now let $L$ be a bounded linear functional on $\mathcal{A}^{1}\left(\mathbb{B}_{*}\right)$. Then $L \circ T^{-1}$ is a bounded linear functional on $\mathcal{E}^{1}(\mathbb{M})$. By Theorem 3.14 there is a function $\widetilde{g} \in \mathcal{E}(\mathbb{M})$ such that $L \circ T^{-1}(\widetilde{f})=\Lambda_{\mathbb{M}}(\widetilde{f}, \widetilde{g})$ for all $\widetilde{f} \in \mathcal{E}^{1}(\mathbb{M})$. But $\widetilde{g}=T g$ for some $g \in \mathcal{B}\left(\mathbb{B}_{*}\right)$. Then for all $f \in \mathcal{A}^{1}\left(\mathbb{B}_{*}\right)$ we have

$$
L(f)=L \circ T^{-1}(T f)=\Lambda_{\mathbb{M}}(T f, T g)=\Lambda_{\mathbb{B}_{*}}(f, g) .
$$

Therefore $L=\Lambda_{\mathbb{B}_{*}}(\cdot, g)$. We also have the uniqueness and the equivalence of the norms. The proof is complete.

Problem 4.3. Theorem 4.10 of [Tim1] shows that the Bloch space of the unit ball can also be characterized by the norm

$$
|f(0)|+\sup _{z \in \mathbb{B}}|\nabla f(z)|\left(1-|z|^{2}\right)
$$

where $\nabla f(z)$ is the complex gradient of the holomorphic function $f$. In the case of $\mathbb{B}_{*}$ it does not seem to be easy to prove the analogue of Theorem 4.10 of [Tim1]. Thus it would be very interesting to find a similar characterization of $\mathcal{B}\left(\mathbb{B}_{*}\right)$ - if it is possible - in terms of the gradient, the norm $N_{*}$ and the weight $|z \bullet z|^{1 / 2}$.

\section{References}

[A] S. Axler, Bergman Spaces and Their Operators, in: Surveys of Some Recent Results in Operator Theory, Vol. I, Res. Notes Math. Ser. 171, Longman, 1988, 1-50. 
[B] D. Békollé, The dual of the Bergman space $A^{1}$ in symmetric Siegel domains of type II, Trans. Amer. Math. Soc. 296 (1986), 607-619.

[Ch] B. R. Choe, Projections, the weighted Bergman spaces and the Bloch space, Proc. Amer. Math. Soc. 108 (1990), 127-136.

[Co] B. Coupet, Le dual des fonctions holomorphes intégrables sur un domaine strictement pseudo-convexe, ibid. 102 (1988), 493-501.

[HP] K. T. Hahn and P. Pflug, On a minimal complex norm that extends the real Euclidean norm, Monatsh. Math. 105 (1988), 107-112.

$[\mathrm{K}] \quad$ K. T. Kim, Automorphism groups of certain domains in $\mathbb{C}^{n}$ with singular boundary, Pacific J. Math. 151 (1991), 54-64.

[KM] S. Krantz and D. Ma, Bloch functions on strongly pseudoconvex domains, Indiana Univ. Math. J. 37 (1988), 145-163.

[L] E. Ligocka, The Bergman projection on harmonic functions, Studia Math. 85 (1987), 255-271.

[M] G. Mengotti, Duality theorems for certain analytic spaces on the minimal ball, Arch. Math. (Basel) 75 (2000), 389-394.

[MY] G. Mengotti and E. H. Youssfi, The weighted Bergman projection and related theory on the minimal ball, Bull. Sci. Math. 123 (1999), 501-525.

[OPY] K. Oeljeklaus, P. Pflug and E. H. Youssfi, The Bergman kernel of the minimal ball and applications, Ann. Inst. Fourier (Grenoble) 47 (1997), 915-928.

[OY] K. Oeljeklaus and E. H. Youssfi, Proper holomorphic mappings and related automorphism groups, J. Geom. Anal. 7 (1997), 623-636.

[PY] P. Pflug and E. H. Youssfi, The Lu Qi-Keng conjecture fails for strongly convex algebraic domains, Arch. Math. (Basel) 71 (1998), 240-245.

[Tem] K. Temgoua, The duals of Bergman spaces in Siegel domains of type II, IMHOTEP J. Afr. Math. Pures Appl. 1 (1997), 41-86.

[Tim1] R. Timoney, Bloch functions in several variables I, Bull. London Math. Soc. 1 (1980), 241-267.

[Tim2] —, Bloch functions in several variables II, J. Reine Angew. Math. 319 (1980), $1-22$.

[Y] E. H. Youssfi, Proper holomorphic lifting of the Bergman and Szegö kernels for some complex domains, preprint, 2001.

[Z1] K. Zhu, Duality and Hankel operators on the Bergman spaces of bounded symmetric domains, J. Funct. Anal. 81 (1988), 260-278.

[Z2] - Operator Theory in Function Spaces, Dekker, 1990.

Institut de Mathématiques

Université de Fribourg

Pérolles, 1700 Fribourg, Switzerland

E-mail: gerald.mengotti@unifr.ch

Received August 7, 2000

Revised version May 2, 2001 